\title{
Principios Biomecánicos en el Tratamiento Quirúrgico de la Esclerosis Idiopática
}

\author{
DR. ENRIQUE IZQUIERDO NUÑEZ
}

Hospital Universitario de Getafe (Madrid)

En el raquis pueden ocurrir dos desviaciones clínicas principales: a) cifosis: desviación del raquis en el plano sagital de concavidad anterior y b) escoliosis: desviación del raquis en los tres planos del espacio. Lordosis es una desviación inversa a la cifosis, es decir, una curvadura de concavidad dorsal en el plano sagital, su existencia aislada es poco frecuente.

La escoliosis se define como una curvadura lateral de la columna en el plano frontal en posición erecta. Se observan con esta curvadura dos cambios importantes. Primero, la curva lateral está en rotación, de tal forma que el cuerpo vertebral está rotado hacia la convexidad de la curva. Segundo, la vértebra situada en el área de máxima desviación lateral está deformada, con una lámina estrecha en la concavidad y con unos pedículos y apófisis tranversas angulados posteriormente en la convexidad. Como el hueso es un tejido vivo, responderá al stress que recae sobre él, con cambios en su estructura, obedeciendo a leyes biológicas. La ley de Wolff discute la interdependencia de la estructura y función y expone que: cada cambio en la forma y o función de los huesos está seguida por cambios en su estructura interna e igualmente define cambios en su configuración externa de acuerdo con leyes matemáticas (Wolff) ${ }^{37}$. Además, estas fuerzas afectan en el crecimiento del hueso. Heuter y Wolkmann ${ }^{12,34}$ propusieron la tesis en la que compresión inhibe el crecimiento mientras que tensión lo estimula.

En el caso de la escoliosis idiopática el crecimiento del raquis, es un factor importante a tener en cuenta para valorar la evolución de la deformidad escoliótica, ya que cuando el crecimiento ha cesado, la progresión de ésta no es muy importante.

El crecimiento vertebral ocurre de una forma similar al de los huesos largos. El aumento de longitud y cambio en la forma, es debido a la actividad de las placas fisarias, no existiendo crecimiento intersticial. En las vértebras no existen epífisis óseas y el crecimiento de cartílago ocurre entre el hueso del cuerpo vertebral y los tejidos del disco intervertebral. Las apófisis en anillo no toman parte en el crecimiento longitudinal ni su oificación tiene relación con este crecimiento. La calcificación y más tarde la osificación de las apófisis en anillo, suceden fuera del plano de la placa de crecimiento fisaria y la fusión se realiza durante varios años, como descubrieron en diferentes etapas y en las diferentes regiones del raquis (Bick y Copel) ${ }^{3}$.

Mientras el estado de osificación de la cresta ilíaca y las apófisis vertebrales en anillo pueden aportar algo acerca del final de la madurez esquelética general, no tienen relación con el crecimiento vertebral ó su cese, debido a que las placas de crecimiento vertebral están presentes hasta la edad de los 25 años, unos diez años más tarde del cese del crecimiento de los miembros inferiores (Inkster) ${ }^{15}$. Esto no implica que continúe el crecimiento vertebral longitudinal significativo hasta este tiempo. Ha sido claramente demostrado que los cuerpos vertebrales son la mitad del tamaño del adulto a la edad de 2 años, con poco crecimiento longitudinal después de los 10 años. Mientras las placas terminales estén abiertas, puede haber cambio en la forma. Esto tiene poca importancia en la columna simétrica, pero puede ser crucial en presencia de escoliosis estructural, debido a que está sujeta a enormes fuerzas asimétricas. Por tanto el efecto en el crecimiento longitudinal es marginal, pero su impacto en la progresión de la escoliosis no debe subestimarse. Esto explica que hallazgos recientes han visto que la mayoría de las curvas idiopáticas progresan hasta esa edad (Hassan y Bierkrein) ${ }^{11}$.

Se han publicado diversos trabajos acerca del cese del crecimiento de la columna vertebral. Risser ${ }^{27}$ demostró que la madurez esquelética del raquis podía ser valorada por la osificación de las apófisis ilíacas y este proceso puede ser cuantificado, dividiendo la cresta ilíaca en cuartos. Risser y Fergusson ${ }^{29}$ publicaron un trabajo en el que concluyen que la progresión de la curva que era de $10^{\circ}$ de media anual, ceso cuando el núcleo de osificación de la cresta llegó a la región ilíaca postero-superior. Sin embargo James ${ }^{18}$ y posteriormente Calvo ${ }^{4}$ observaron que el crecimiento vertebral no cesó cuando las apófisis ilíacas habían completado su osificación. Tupman publicó que le crecimiento del fémur y la tibia en niñas, cesa a la edad de 14,4 y 15,5 respectivamente, mientras que el tronco no había cesado de crecer después de los 16 años.

Ponseti y Friedman ${ }^{26}$ publicaron que curvas escolióticas progresaron cerca de un grado por año en la vida adulta y el aumento medio en las curvas después de la madurez esquelética era $15^{\circ}$ y que las curvas de más de $60^{\circ}$ eran las que más se deterioraban.

Dickson y Leatherman ${ }^{7}$ encontraron 10 casos de escoliosis idiopática que tenían radiografias hechas después de llegar a la madurez esquelética y también algunos años más tarde. En estos casos la curva media inicial de $44^{\circ}$ había progresado a 60 , después de un intervalo medio de 6 años. Una curva había aumentado desde 40 a la edad de 20 años, a $95^{\circ}$ a la edad de 33 años.

Stagnara y cols. 32 vieron que adultos podían perder hasta 24 centímetros en altura como resultado de la progresión de su escoliosis. 

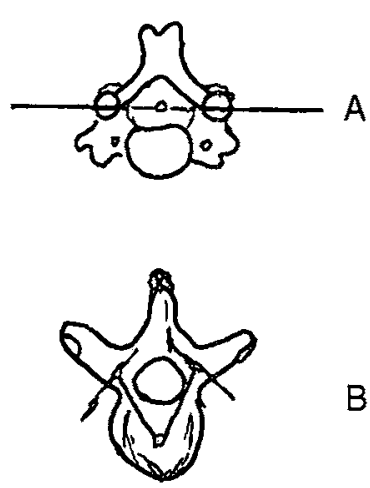

$B$

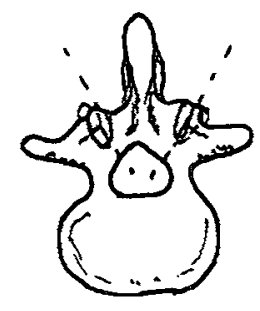

C

Fig. 1. La inclinación de las carillas articulares determina el eje de rotación. En las regiones cervical (A) y lumbar (C), el eje es posterior al cuerpo vertebral, mientras que en el región torácica (B), es anterior al cuerpo vertebral.

En el momento en que se asume la posición erecta aparecen cuatro curvas primarias en el plano sagital: lordosis en las regiones cervicales y lumbares y cifosis en las torácicas y sacras. En el plano coronal la columna es recta aunque a veces hay una mínima curva torácica. El eje de rotación vertebral, determinado en gran parte por la orientación de las carillas articulares (Fig. 1) discurre a través del raquis, situándose en un plano posterior en la lordosis cervical y lumbar, y anterior en la columna torácica y sacra (Fig, 3). El centro de gravedad del cuerpo erecto, se sitúa ventral a toda la columna vertebral y la tendencia en una columna sin soportes, es a flexionarse hacia adelante. Una curva lordótica, es inestable a la flexión ventral, originando que la columna se acomode con dificultad a uno y otro lado sin rotar. En contraste, en una curva cifótica, no sucede este movimiento por estar el eje de rotación situado ventralmente, produciéndose si acaso, una mínima rotación; aquella que está permitida por los límites de elasticidad de la orientación de las carillas articulares.

En áreas lordóticas fisiológicas existen una serie de mecanismos para resistir la rotación bajo circunstancias normales: primero una gran amplitud de flexión intersegmentaria, por lo que la columna lumbar llega a ser cifótica antes de que se alcance el límite de flexión; segundo, la configuración de las vertebras cervicales y lumbares. Estas al tener un cuerpo ancho transversalmente y unos procesos espinosos largos orientados hacia arriba, se forma un prisma de base anterior que al flexionar ventralmente (es decir hacia la base del prisma), lo hará sin que exista inestabilidad rotacional (Fig. 2).

En la región torácica los cuerpos ventrales con las apófisis espinosas adoptan una forma de corazón

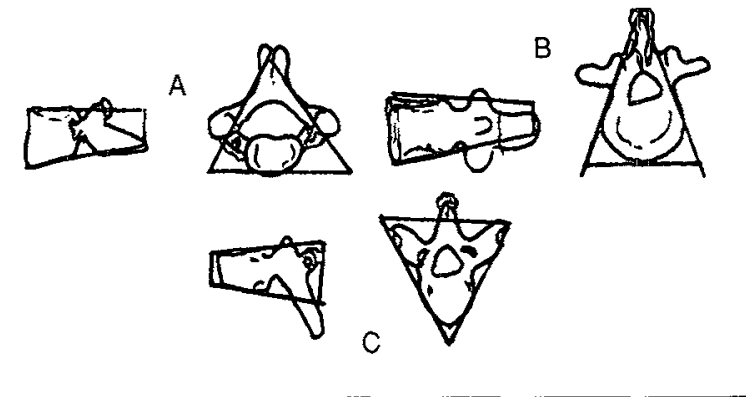

Fig. 2. En las regiones cervical (A) y lumbar (B), estas vértebras lordóticas se modelan en su sección transversal igual que un prisma con su base situada anteriormente. En la región torácica $(\mathrm{C})$, estas vértebras cifóticas se modelan igual que un prisma con su ápex situado anteriormente.

(Fig. 2). Las apófisis espinosas son largas, aplanadas y están situadas en la parte posterior del raquis. Forman un prisma con su ápex anterior, que hace que en flexión estas vertebras torácicas sean rotacionalmente inestables; como elemento añadido de inestabilidad, hay que considerar el acuñamiento asimétrico de los cuerpos vertebrales debido a la aorta. La existencia de la cifosis torácica protege a la columna contra la rotación, pero como hay poca flexión intersegmentaria, cualquier pérdida de cifosis producirá una curvadura rotacional.

Para el estudio biomecánico de la columna se han diseñado distintos modelos. Rogers ${ }^{30}$ utilizó vástagos flexibles e investigó los factores que estaban en relación con la causa de la curva. El único hallazgo positivo era, que se jproduce una curva cuando existe un desequili-

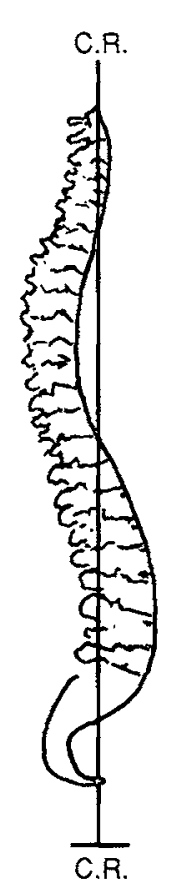

Fig. 3. El eje de rotación de la columna es consecuencia de la inclinación de las carillas articulares. 
brio en los músculos posteriores (erector de la columna). Lindahl y Reader ${ }^{21}$ utilizaron un modelo matemático y demostraron que la columna puede flexionarse sin rotación si los extremos no están fijos. Rotación ocurre si los extremos están sujetos y la rotación máxima ocurre en el centro de la curva. Postularon que la escoliosis idiopática era debido a un freno o sujección posterolateral debido a una contractura en esta área o porque los ligamentos están ligeramente tensos y como consecuencia de ello se producirá una curva con crecimiento en el lado opuesto.

Asmussen ${ }^{1}$ aplicó la ley de Euler a una columna torsionada, basado en un principio de ingenieria que se define por la fórmula:

$$
P(\text { crit })=C\left(E I / L^{2}\right)
$$

donde p (crit) es la carga crítica, C la condición final, E el módulo de elasticidad, I el momento de inercia d ela sección transversal con respecto a la dirección de carga y el plano de la curvadura y L la longitud de la columna.

El aumento de la longitud reduce la carga crítica haciendo que la columna falle. También inciden en ello la dirección de dicha carga, la sección transversal, la simetria y la resistencia del material de la columna.

Por este motivo se puede observar que la carga crítica ejercida sobre la columna, en la región torácica (vértebra en forma de corazón), cuando se flexiona ventralmente, se torsiona con facilidad y ante la asimetria por la acción de la aorta se pueda producir una curva lateral.

En los tejidos vivos la progresión de la deformidad sucede igual que en el material inerte. La recuperación de los efectos de carga toma un tiempo finito, siguiendo el efecto histéresis. Si la carga se aplica antes de que se complete la recuperación se producirá una deformación. A este fenómeno se suma el efecto de la ley de HueterWolkmann si el tejido está en crecimiento. En el caso de la columna torácica el borde anterior crece más rapidamente y el posterior más lentamente, es decir, se produce un acuñamiento posterior y como consecuencia de ello la evolución hacia una lordosis. En lordosis, las facetas del raquis torácico no están alineadas para permitir ninguna rotación, por lo que al producirse dicha rotación, aquellas se deformarán. La carga siguiente deformante aplicada antes de que se consiga la recuperación a la carga previa (efecto onda histéresis), unido al efecto de aumento de la carga sobre tejidos en crecimiento en el lado de la compresión y de disminución en el lado de la tensión, hara que a la alteración en la velocidad de crecimiento se añada el aumento de la deformidad. Esta deformidad esencialmente lordótica hace que la porción posterior de la columna deformada sea más corta que la anterior. La carga sobre la epífisis del lado del cuerpo vertebral (que está ahora dirigida más ventralmente), aumenta conforme progresa la rotación y como está todavía detrás del centro de gravedad se producirá una deformación lateral por acuñamiento de la vértebra. Se produce por tanto la escoliosis una deformidad triplanar: lordosis, rotación y acuñamiento lateral de las vértebras afectadas.
Dickson y cols. ${ }^{6}$ hicieron radiografías a cinco especímenes con escoliosis en proyecciones antero-posterior y lateral; midieron un ángulo de Cobb medio de $67^{\circ}$ de escoliosis y $76^{\circ}$ de cifosis, pero en proyecciones reales la escoliosis aumentaba a $90^{\circ}$ en proyección A-P y en la proyección lateral existía una lordosis media de $3^{\circ}$ en el ápex de la curva. Realizando una tomografía axial computerizada demostraron que cada vértebra de la curva tenía un ángulo distinto de rotación excepto las vértebras $\mathrm{T}_{7}$ y $\mathrm{T}_{8}$ que presentaban un ángulo de $30^{\circ}$. La tomografía computerizada vertical demostró que no existía cifosis.

La curvadura raquídea en el plano sagital cambia durante el crecimiento en niños normales, de tal forma que la cifosis torácica se reduce desde la edad de 8 a 14 años, siendo mínima a la edad de 12 años, coincidente con el periodo de maduración de las niñas que es dos años antes que los niños y es cuando se encuentran en un pico de crecimiento álgido. Esto puede explicar el mayor potencial de progresión de escoliosis latentes en las niñas. Cuando la cifosis torácica es mínima en los niños, su velocidad de crecimiento es constante; mientras que en la adolescencia tardía, cuando tienen su pico de crecimiento máximo, la cifosis torácica se encuentra en su mayor magnitud. Esto explicaría porqué los niños están predispuestos a desarrollar más facilmente la enfermedad de Scheuermann.

\section{TRATAMIENTO}

Para la mayoria de los pacientes con escoliosis idiopáticas observación será el único tratamiento necesario.

El objetivo en el tratamiento de la escoliosis es prevenir la progresión de la curva, por lo cual es preciso conocer la historia natural de esa curva, aunque desgraciadamente saber que cuva va a progresar no se conoce. En curvas pequeñas inferiores a $40^{\circ}$ un corsé puede controlar la progresión de éstas, sin embargo curvas mayores serán susceptibles de tratamiento quirúrgico.

\section{Los objetivos de la cirugia de la escoliosis son:}

$1^{\circ}$. Detener la progresión o empeoramiento de la deformidad y por tanto evitar la secuela de la alteración de la función pulmonar y dolor.

$2^{\circ}$. Conseguir la corrección máxima en los tres planos, con un mínimo de complicaciones neurológicas.

$3^{\circ}$. Obtener un tronco y torso equilibrado.

$4^{\circ}$. Conseguir que los tres primeros objetivos sean permanentes por medio de una artrodesis sólida.

Ciertos principios biomecánicos se aplican a todos los tipos de tratamiento. La columna es una unión de estructuras sólidas (vértebras) conectadas por tejidos móviles (discos y ligamentos) y funciona como una estructura biológica viscoelástica.

Suceden dos fenómenos debido a esta conducta viscoelástica: Deslizamiento y relajación.

Deslizamiento se define como una deformación que sigue a la carga inicial en un material y sucede como una función de tiempo sin carga adicional. Por lo tanto 


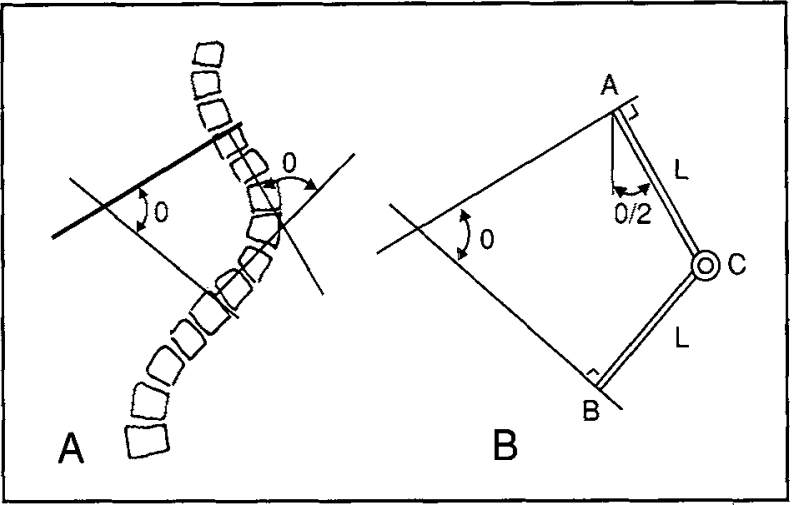

Fig. 4. Modelo para la corrección de la escoliosis. (A) Curva escoliótica de 0. (B) Modelo simplificado con dos brazos AC y CB conectados por un muelle torsional en $\mathrm{C}$ forman un ángulo $0^{\circ}$. (Tomado de White y Panjabi).

cuando una fuerza correctora se aplica a una deformidad, hay una correción con alargamiento del segmento de la columna. La corrección subsiguiente que ocurre debido a la misma fuerza sobre un periodo de tiempo es debido al deslizamiento con una carga se aplica a un material viscoelástico y la deformación se mantiene constante, la disminución de la carga subsiguiente observada al cabo de tiempo es la relajación. Clínicamente una fuerza se usa para corregir una deformidad vertebral pero con el tiempo la corrección no cambia y la fuerza disminuye.

Se pueden aplicar diferentes fuerzas correctoras a una columna escoliótica, por lo que se requiere una simulación y evaluación de los efectos como ya se ha visto en un modelo complejo tridimensional. White y Panjabi ${ }^{35,36}$ vieron que ciertos principios básicos se pueden ilustrar con un modelo simplificado (Fig. 4). La columna escoliótica está representada por dos brazos $\mathrm{AC}$ y $\mathrm{CB}$ con una longitud L. Los brazos están conectados en este modelo por un muelle torsionado y están orientados para simular una curva de ángulo $0^{\circ}$ medio por el método de Cobb. Se puede aplicar una fuerza axial, transversa o combinada a la columna para corregir la deformidad. La corrección que se obtiene no es debida a una simple tensión en la columna sino más bien a momentos de flexión en cada nivel discal intervertebral, y la combinación de éstos producirá la corrección de la deformidad angular.

Si se aplica una fuerza axial $\mathrm{F}$ a los dos extremos de los dos brazos en los puntos A y B (Fig. 5), se producirá elongación y estiramiento. El movimiento corrector en el ápex de la curva está representado por la fuerza $\mathrm{F}$ multiplicado por la distancia perpendicular al ápex de la curva $\mathrm{D}$. Al aumentar la curva, la distancia $\mathrm{D}$ aumenta; y además el radio de la curva disminuye, formando lo que se ha denominado curva de radio corto. Al aumentar D, la capacidad correctora de la fuerza aumenta. En otras palabras, la capacidad de corrección de una fuerza axial aumenta con la gravedad de la curva.

La fuerza transversal cuando se aplica en el ápex de la curva se muestra en la (Fig. 6). La fuerza F resulta en un momento corrector igual a la fuerza en el final de la

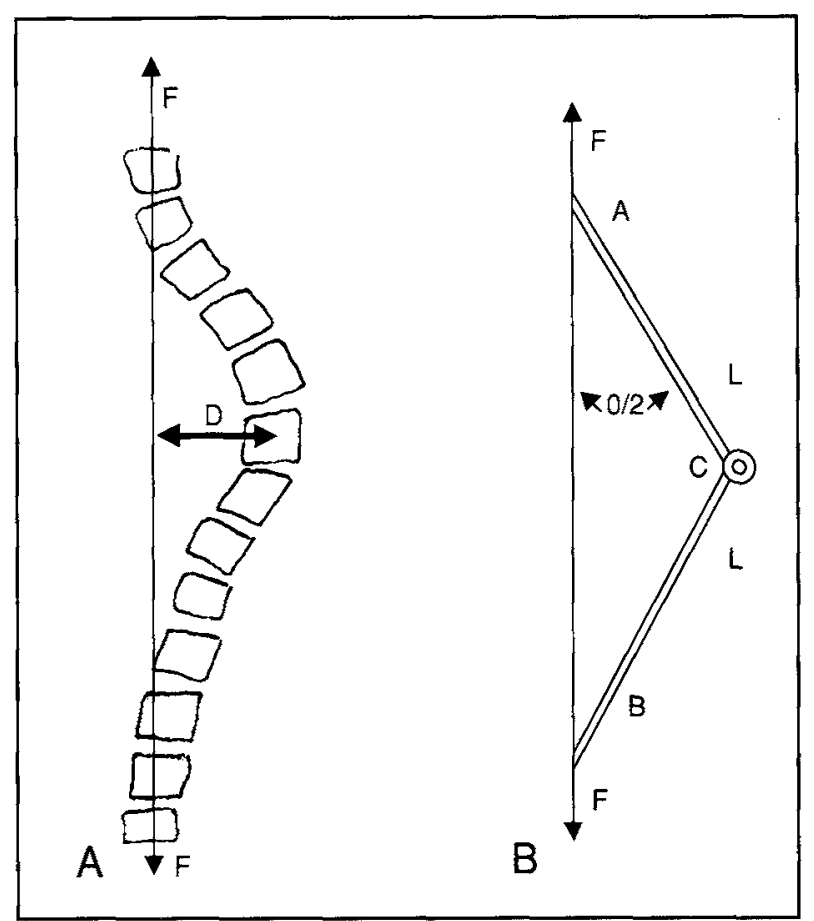

Fig. 5. Aplicación de una fuerza axial F a una curva escoliótica, siendo $\mathrm{D}$ la distancia desde la perpendicular de la fuerza al ápex de la curva. (Tomado de White y Panjabi).

curva (F/2) multiplicado por la distancia perpendicular D de este final al ápex de la curva. Con curvas más pequeñas la distancia $\mathrm{D}$ aumenta, resultando una curva de radio largo. La distancia $\mathrm{D}$ siminuye según la deformidad aumenta; la capacidad correctora de una fuerza transversal por tanto disminuye con el aumento de la curva. Una fuerza axial es más eficiente para la corrección de curvas de radio corto, mientras que una fuerza tranversa corrige las curvas de radio largo.

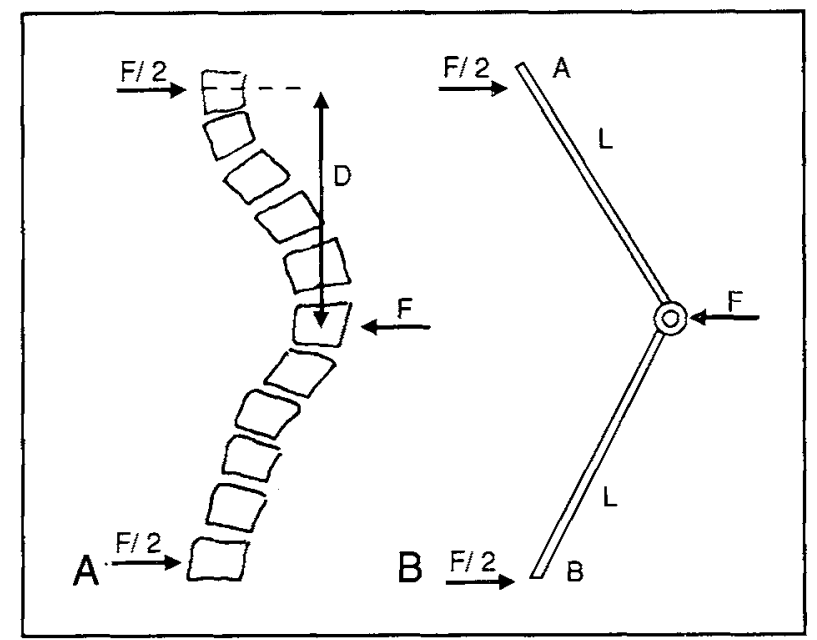

Fig. 6. La aplicación de una fuerza transversa $F$ a una curva escoliótica resulta en una fuerza correctora $F / 2$ en los extremos de la curva. D es la distancia desde la perpendicular de F/2 al ápex de la curva. (A) Columna escoliótica sometida a una fuerza transversa. (B) Modelo simplificado de una columna escoliótica sometida a ua fuerza transversa. (Tomado de White y Panjabi). 
Estas fuerzas correctoras pueden actuar solar o conjuntamente, y en general suele ser más beneficioso una combinación de fuerzas transversales y axiales. Una comparación de la eficacia de estas fuerzas la realizaron White y Panjabi. Se calculó el momento flexión relativo en el espacio apical discal y se obtuvo más corrección con el momento de flexión aumentado. La Fig. 7 muestra estos resultados gráficamente, con la deformidad angular medida en grados por el método de Cobb. En el eje vertical el factor $\mathrm{M} / \mathrm{FL}$ representa el momento corrector relativo, mientras que $\mathrm{M}$ es el momento de fuerza corrector, F es la fuerza correctora, y L la distancia $\mathrm{AC}$ de la Fig.; los valores $\mathrm{F}$ y $\mathrm{L}$ son los mismos para los tres gráficos. Se ha visto que el momento corrector de una fuerza axial aumenta según la deformidad angular aumenta, mientras que la carga tranversa disminuye. Las dos curvas se cruzan en una deformidad angular de $53^{\circ}$, indicando que una fuerza transversa es más eficiente debajo de este valor mientras que una fuerza axial es más eficiente por encima. El gráfico también muestra que una fuerza combinada teoricamente tiene un momento corrector relativo mejor.

Otra fuerza correctora que se puede aplicar a una curvadura espinal es una de compresión axial aplicada en la convexidad de la curva. Esta fuerza se puede aplicar indistintamente anterior 0 posterior.

Durante los últimos años se han desarrollado diversas técnicas de corrección quirúrgica de las escoliosis. Harrington ${ }^{10}$ dio un paso muy importante al desarrollat una barra distractora sobre dos ganchos. Depende de fuerzas de tensión aplicadas al lado cóncavo de la curva. Varios estudios demostraron que el método de Harrington usado correctamente corregía alrededor del $60 \%$ de la curva escoliótica, observandose una pérdida de corrección de un $10 \%$, una fusión sólida en el $96 \%$ de los casos, y un número de complicaciones neurológicas aceptablemente bajo (King y cols.20, Mc Ewen y cols. ${ }^{24}$ ).

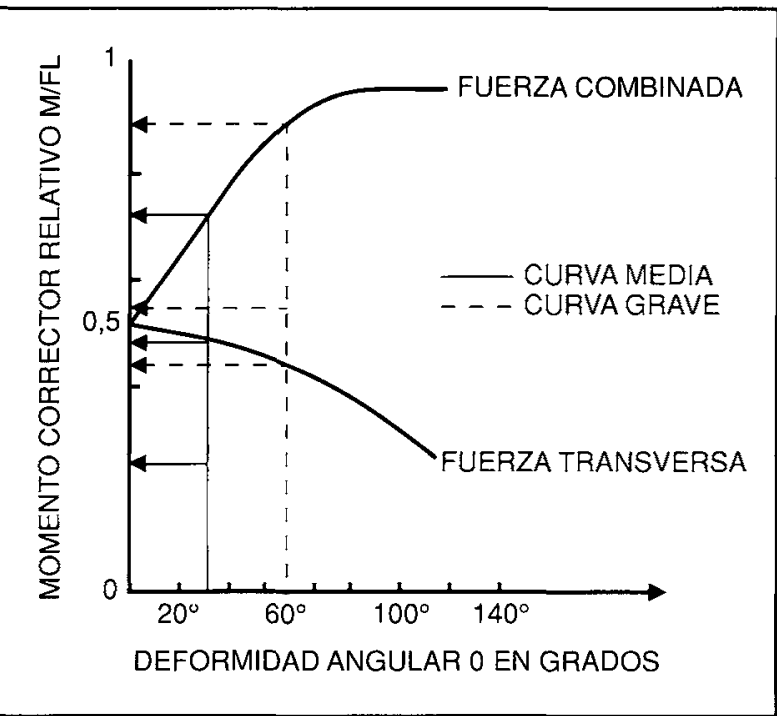

Fig. 7. Representación gráfica del momento corrector relativo M/FL como una función de la diformidad angular en grados. (Tomado de White y Panjabi).
Sin embargo hay dos desventajas con el método de Harrington:

$1^{\circ}$. La distracción es un sistema efectivo en el plano frontal pero practicamente nulo en el plano axial y además puede aplanar el contorno sagital (Ginsburg y cols. ${ }^{9}$ )

$2^{\circ}$. Biomecanicamente corrige la curva en solo dos puntos de apoyo, situados en los extremos. Debido a que no hay puntos de anclaje intermedios puede haber pérdida de corrección, por lo que es necesario utilizar un corsé de yeso en el post-operatorio.

Hasta 1978 ha sido la única instrumentación por vía posterior utilizada para la corrección de la curva escoliótica, aunque se hicieron algunas modificaciones a ella, como los vástagos cuadrados de Moe, para evitar la rotación de éstos sobre el gancho o el sistema DDT de Cotrel, que utiliza un tercer punto de apoyo a nivel de las vértebras del ápex de la curva escoliótica, consiguiendo de esta manera un montaje más sólido.

En 1978 Luque $^{23}$ obtiene la corrección de las curvas por una acción esencialmente en el plano transverso, y consigue una fijación segmentaria sólida, por medio de alambres sublaminares fijados a dos barras paralelas en $\mathrm{L}$, que permite no utilizar corsé como soporte post-operatorio.

El sistema combinado Harrington-Luque (HarriLuque), aplicado por la escuela de Minnesota, combina métodos de distracción y fijación segmentaria.

El avance más importante en el tratamiento de la escoliosis ha sido la apreciación conceptual de la deformidad. La escoliosis se trata como una deformidad multiplanar, por lo que admeás de corregirla en el plano frontal se debe hacer en el plano axial y sagital.

La importancia fisiológica de tener un contorno sagital normal se reconoce actualmente. La lordosis torácica o hipocifosis (espalda plana), tiene mayor efecto adverso sobre la fisiologia pulmonar que la curva en el plano frontal. También, el aplanamiento de la lordosis normal produce con el tiempo dolor lumbar (Ginsburg y cols. ${ }^{9}$ ).

Otro avance, es el mejor conocimiento de los principios biomecánicos. Con los nuevos sistemas, hay múltiples puntos de anclaje, dando una mayor estabilidad, aumento de la resistencia en la fatiga del implante y menor incidencia de seudoartrosis en la masa de fusión.

El método más moderno y conceptualmente más atractivo, fue desarrollado por Cotrel y Dubousset ${ }^{5}$. El principio del sistema Cotrel-Dubousset es que la corrección se consigue contorneando los vástagos a la curva deseada en el plano sagital. Se inserta el primer vástago en unos ganchos previamente situados, se contornea éste en la misma dirección de la curva escoliótica, y una vez insertado el vástago en todos los ganchos se rota 90 grados. Rotando el vástago hasta el perfil recto, corrige en el plano coronal, ejerce al mismo tiempo un alineamiento en el plano sagital y una corrección en plano axial (entre un 25 y un $33 \%$ ), contribuyendo a una mejoría de la corrección de la gibosidad costal. 
Test mecánicos han demostrado que es el sistema más rígido y estable de todos los estudiados (Schufflebarger ${ }^{31}$ ). Tiene el peligro de penetrar en el canal vertebral con el gancho durante la maniobra de rotación. Es un sistema complejo que precisa de tiempo para el aprendizaje. Seleccionar el nivel vertebral distal a fusionar, es importante, ya que se puede producir un desequilibrio del raquis, aunque con el tiempo este desequilibrio se autocorrige (Izquierdo y cols. ${ }^{16}$ ). Esto se ha visto principalmente en las curvas de tipo King II, y para estos casos se ha enfatizado la importancia de estudiar el plano satinal radiográficamente, para detectar la transición entre las curvas torácica y lumbar, antes de seleccionar los niveles de instrumentar y nunca acabar la instrumentación en la unión toraco-lumbar. También puede producir cifosis en el extremo final de la instrumentación.

Otro problema con la instrumentación CotrelDubousset, es que es voluminosa, puede dar problemas de escaras e infección, es una instrumentación costosa y es tres veces más peligrosa neurológicamente que la técnica de Harrington, siendo la frecuencia de complicaciones neurológicas alrededor del $3 \%$.

La indicación de esta instrumentación es en los cinco tipos de curvas torácicas según King ${ }^{20}$.

El gran paso para la corrección de las curvas vertebrales toraco-lumbares o lumbares, por via anterior, se realizó en 1.969 por Dwyer ${ }^{8}$ utilizando un sistema de tornillos y cables, que realizan compresión de los cuerpos vertebrales previa extirpación de discos e introducción de injerto de la costilla extirpada.

Zielke y cols. ${ }^{38}$, modificaron en 1.976 la técnica de Dwyer, usando una barra roscada en lugar del cable y además aportando un dispositivo para corregir la rotación de la curva instrumentada. La barra roscada de Zielke es superior biomecánicamente al cable de Dwyer.

La corrección que se obtiene con esta instrumentación en el plano anteroposterior es alrededor de un $80 \%$ (Moe y cols.25, Kaneda y cols. ${ }^{19}$, Zielke y cols..$^{38}$, Izquierdo y cols. ${ }^{16}$ ).

La corrección de la rotación de la vértebra ápex de la curva lumbar medida con el torsiómetro de Perdriolle, está alrededor de un $45 \%$ (Kaneda y cols. ${ }^{19}$, Luk y cols. ${ }^{22}$ ), siendo muy superiores estos valores a los obtenidos con la técnica de Harrington, que están alrededor de un $20 \%$ (Luk y cols. ${ }^{22}$, Hsu y cols. ${ }^{13}$ ). Por ello, es la instrumentación idónea para la corrección de las curvas toracolumbares o lumbares.

\section{BIBLIOGRAFIA}

1. Asmussen, E.: The weight carrying function of the human spine. Acta Orthop. Scand. 29, 276, 1960.

2. Bick, E., Copel, J.: Longitudinal growth of the human vertebra: a contribution to human osteogeny. J. Bone Joint Surg.. 32 A, 803, 1950.

3. Bick, E., Copel, J.: The ring apophysis of the human vertebra. Contribution to human osteogeny. J. Bone Joint Surg. 31 A, 787, 1951.

4. Calvo, L.: Observations on the growth of the female adolescent spine and its relations to scoliosis. Clin. Orthop. 10, 40, 1957.

5. Cotrel, Y., Dubousset, J.: Nouvelle Technique d'osteosynthese rachidienne segmentaire par voi posteriere. Rev. Chir. Orth. 70, 489, 1984.

6. Dickson, R., Lawton, J., Archer, I., Butt, W.: The pathogenesis of idiopathic scoliosis: biplanar spinal asymmetry. J. Bone Joint Surg. 66 B,
$8,1984$.

7. Dickson, R. Leatherman, K.: Spinal deformity in adults: Changing concepts. J. Bone Joint Surg. 58 A, 729, 1976.

8. Dwyer, A.: An anterior aproach to scoliosis. A preliminar report. Clin. orthop. 62, 192, 1969.

9. Ginsburg, H., Goldstein, L., Robinson, S., Haake, P., Davanney, J., Chand., Suk, E.: Back pain in postoperative idiopathic scoliosis. Orthop. Trans. 3, 50, 1973 .

10. Harrington, $P$.: Treatment of scoliosis correction and internal fixation by spine instrumentation. J. Bone Joint Surg. 44 A, 591, 1962.

11. Hassan, I., Bjekrein, I.: Progression in idiopathic scoliosis after conservative treatment. Acta Orthop. Scand. 54, 88, 1983.

12. Henter, C.: Anatomische Studien an den Extremitaten neugeborener und erwachsener. Wirchows Arch. A, vol. 25, 575, 1870.

13. Hsu, J., Zucherman, J., Tang, S., Leong, J.: Dwyer instrumentation in the treatment of idiopathic scoliosis. J. Bone Joint Surg. 46 B, 536, 1982.

14.?

15. Inkster, R.: Osteology in: Brasch, J., ed. Cunningham's test book of anatomy. 9 th. ed. London Oxford University Press, 105, 1951.

16. Izquierdo, E., Fernández y Fernández Arroyo, A., Pérez Núñez, M!I!, Fernández de Valderrama, J.: tratamiento de la escoliosis idiopática tipo K II con instrumentación C-D. Rev. Ortop. Traum. (en prensa).

$17 . ?$

18. James, J.: Idiopathic scoliosis. The prognosis, diagnosis and operative indications related to curve patterns and the age at onset. J. Bone Joint Surg. 36 B, 36, 1954.

19. Kaneda, W., Fijiya, N., Satoh, S.: Results with Zielke instrumentation for idiopathic thoracolumbar and lumbar scoliosis. Clin. Orthop. $195,295,1986$.

20. King, H., Moe, J., Bradford, D.: The selection of fusion levels in thoracic idiopahic scoliosis. J. Bone Joint Surg. 46 A, 1303, 1983

21. Lindahl, O., Raeder, E.: Mechanical Analysis of Forces Involver in Idiopathic Scoliosis. Acta Orthop. Sand. 32, 27, 1962.

22. Luk, K., Leong, J., Hsu, L.: The comparative results of treatment in idiopathic thoracolumbar and lumbar scoliosis using the Harrington, Dwyer and Zielke instrumentations. Spine 14, 275, 1989.

23. Luque, E., Cardoso, A.: Segmental correction of scoliosisi with rigid internal fixation. Annual meeting Scoliosis Research Society. Ottawa, Ontario, Canada, September 1976.

24. Mc Ewen, G., Bunnel, W., Sriran, K.: Acute neurological complications in the treatment of scoliosis. J. Bone Joint Surg. 55 A, 404, 1973.

25. Moe, J., Purcell, G., Bradford, D.: Zielke instrumentations (VDS) for the correction of spinal curvature. Clin. Orthop. 180, 133, 1983.

26. Ponseti, I., Friedman, B.: Prognosis in idiopathic scoliosis. J. Bone Joint Surg. 32 a, 381, 1950.

27. Risser, J.: The iliac apophysis:an invaluable sign in the management of scoliosis. Clin. Orthop. II-III 9, 1958.

28. Risser, j.: Scoliosis; its prognosis. J. Bone Joint Surg. 18, 637, 1936.

29. Risser J., Fergusson, A.: Scoliosis: its prognosis. J. Bone Joint Surg. $18,637,1936$.

30. Rogers, S.: Mechanics of Scoliosis. Arch. Surg. 26, 962, 1933.

31. Schufflebarger, H.: Biomecanical studies with C-D instrumentation on fracture model, 4th Annual Symposium on C-D instrumentation. Miami. Florida 1987.

32. Stagnara, P., Gonan, G., Fauchet, P.: Surgical treatment of idiopathic rigid lumbar scoliosis in the adult. In: Dickson, R., Bradford, D. eds. Management of spinal deformities. Butterworths International Medical Reviews Orthopaedics 2, London etc., Butterworths 303, 1984

33. Tupman, G.: A study of bone growth in normal children and its relationship to skeletal maturation. J. Bone Joint Surg. 44 B, 42, 1962.

34. Volkmann Chirurgische Erfahrungen ueber Knorchenverbiegungen und knochenwachstum, Arch. Pathol. Anat. 24, 512, 1862.

35. White, A., Panjabi, M.: Clinical Biomechanics of the Spine Lippincott, Philadelfia, 1978.

36. White, A., Panjabi, M.: Clinical Biomechanics of Scoliosis, Clin. Orthop., 118, 100, 1976.

37. Wolff, J.: Uber die innere Architecture der Knochen und ihre Bedeutun für die Frage von Knochenwachstum, Vircows Arch. A, vol. $25,589,1970$.

38. Zielke, K., Stunkat, R., Beaujeau, F.: Ventral derrotations spondilodese. Arch. Orthop. Unfallchir. 85, 257, 1976. 